\title{
The Efficacy of Cognitive Behavioral Therapy for Insomnia in Patients with Chronic Pain
}

\author{
Carla R. Jungquist, RN, $\mathrm{PhD}^{1,2,3}$, Chris O'Brien, MSN ${ }^{1,3}$, Sara Matteson-Rusby, PsyD ${ }^{3}$, \\ Michael T. Smith, $\mathrm{PhD}^{4}$, Wilfred R. Pigeon, $\mathrm{PhD}^{3}$, Yinglin $\mathrm{Xia}^{5}$, Naiji Lu ${ }^{5}$, and Michael L. Perlis, \\ $\mathrm{PhD}^{6}$ \\ ${ }^{1}$ University of Rochester, School of Nursing \\ 2University of Rochester, Department of Anesthesiology \\ ${ }^{3}$ University of Rochester, Sleep and Neurophysiology Research Laboratory \\ 4John Hopkins University, Department of Psychiatry and Behavioral Sciences \\ ${ }^{5}$ University of Rochester, Department of Biostatistics \\ ${ }^{6}$ University of Pennsylvania, Department of Psychiatry
}

\section{Introduction}

In recent years there has been a growing interest in the veracity of the concept of "Secondary Insomnia" and the assumption that such forms of sleep continuity disturbance are likely to be more resistant to treatment than Primary Insomnia. This challenge has largely occurred at a theoretical level. Lichstein et al. $(1,2)$, have argued that it is nearly impossible to substantiate that insomnia is truly secondary and thus the distinction has little nosological value and should not be used to dictate when targeted treatment is warranted. This point of view is further buttressed by the Spielman Model $(3,4)$ which clearly suggests that chronic insomnia is maintained, irrespective of the original precipitants, by a common set of perpetuating factors which are largely cognitive and behavioral in nature. When taken together, these lines of thought suggest that treatments for Primary Insomnia should be effective for what is now considered "co-morbid insomnia" $(5,6)$. One very plausible exception to this perspective is in conditions where the comorbid illness may act as both a precipitating and perpetuating factor. This scenario is likely to be the case with insomnia comorbid with chronic pain. The unremitting nature of chronic pain may directly contribute to acute and chronic sleep initiation and maintenance problems via a form of hyperarousal that comes with the experience of pain. To date, three trials with Cognitive Behavioral Therapy for Insomnia (CBT-I) ${ }^{\mathrm{i}}$ have been conducted in the context of chronic pain (7-9). The studies are briefly summarized below (see also Table 1).

\footnotetext{
Corresponding author(s): Carla Jungquist, RN, PhD, University of Rochester, Department of Anesthesiology, 601 Elmwood Avenue, Box 604, Rochester, NY 14642, 585-275-3524, 585-473-5007 (fax), Carla_Jungquist@URMC.Rochester.edu; Michael Perlis, PhD, Associate Professor, University of Pennsylvania, Department of Psychiatry, Blockley Hall, Philadelphia, PA 19104-6021215-746-3577, 270- 512-9828 (fax), mperlis@ mail.med.upenn.edu.

Publisher's Disclaimer: This is a PDF file of an unedited manuscript that has been accepted for publication. As a service to our customers we are providing this early version of the manuscript. The manuscript will undergo copyediting, typesetting, and review of the resulting proof before it is published in its final citable form. Please note that during the production process errors may be discovered which could affect the content, and all legal disclaimers that apply to the journal pertain.

iThe use of "CBT-I" as an acronym has the heuristic advantage of distinguishing this form of therapy from others forms of CBT.
} 
The first study of CBT-I in chronic pain was conducted with 60 chronic pain patients (8). Thirty-two of the subjects were randomized to group CBT-I and 28 were randomized to a selfmonitoring/waiting-list control condition. The second study was conducted with 47 fibromyalgia patients who received individual therapy with CBT-I $(n=18)$, sleep hygiene $(\mathrm{n}=18)$, or treatment-as-usual for fibromyalgia $(\mathrm{n}=11)(9)$. The third study was conducted in 92 elderly patients with insomnia occurring in association with one of three conditions: chronic pain due to osteoarthritis, chronic obstructive lung disease, or coronary artery disease. In this study, the effectiveness of CBT-I was compared to a control condition consisting of stress management (7). All three of the studies included Sleep Restriction and Stimulus Control as the main components of their CBT-I regimens; the Currie et al. and Rybarczyk et al. studies utilized group formats, and only one study (Edinger et al.) had treatment conducted by expert therapists.

In the first study (Currie et al.), significant group differences were observed for self reported sleep latency and wake after sleep onset. CBT-I was not associated with significant group differences in pain severity, although the mean changes were in a positive direction and the effects appeared to be in the moderate range. The pain reduction effect size (pre-to-post) at 3 months for CBT was .56 (moderate) compared to 0.15 for waitlist control $(\mathrm{p}=0.12)(8)$. In the second study (Edinger et al.), significant group differences were observed for self reported sleep latency, total wake time, and sleep efficiency. A trend ( $\mathrm{p}=0.07)$ was evident for wake after sleep onset. CBT-I was not associated with significant changes in pain measures but here again there was a modest mean pre-to-post change with a moderate to large corresponding effect size (0.88). In the third study (Rybarczyk et al.), the three medical conditions responded to CBT-I in a comparable fashion and the aggregate of the samples significantly differed from the control condition for self reported sleep latency, sleep efficiency, and wake after onset. Evaluating the effect of CBT-I on pain was not a focus of this study.

Taken together, these studies support the notion that CBT-I may be successfully utilized with insomnia that occurs co-morbid with chronic pain. Further, two of the three studies provide data (when viewed as effect sizes) that suggest that CBT-I may also confer some clinical benefit with respect to pain.

The purpose of the present study was to 1) garner additional evidence regarding the efficacy of CBT-I in treating insomnia in the context of chronic pain and 2) assess whether CBT-I is associated with clinical changes with respect to pain severity and/or pain interference (daytime function). In the case of the first goal, our intent was to deploy a form of CBT-I that was more rigorous than that provided in the prior studies. In the present study, the intervention was delivered as individual therapy where treatment was conducted according to the specifications of a published treatment manual by a therapist who received intensive training and extensive supervision. In the case of the second goal, a broader measurement strategy was utilized to evaluate pain responses in a homogenous sample of chronic pain patients. In the present study, three measures were used to assess pain: the Pain Disability Index (PDI), The Multidimensional Pain Inventory (MPI) and a Likert scale score on the sleep/pain diaries.

\section{Methods}

This study was approved by the Research Subjects Review Board (RSRB) of the University of Rochester. All subjects signed informed consent before engaging in any research activities.

\section{Design}

Patients with insomnia comorbid with chronic pain were recruited from the community and local pain treatment clinics to participate in parallel-groups, randomized, single blind trial of CBT-I with a contact/measurement control condition. 


\section{Inclusion Criteria}

Age 25 or older (to avoid delayed phase disorder); chronic (> 6 months) non-malignant pain originating in the spine; insomnia reported as originating after, and/or aggravated by, the pain condition; insomnia (defined as $>30$ minutes sleep latency and/or minutes awake after sleep onset for $>3$ days/wk for $>6$ months); preferred sleep phase between $10 \mathrm{pm}$ and $8 \mathrm{am}$ to avoid sleep phase disorders and shift workers; AHI < 10; no evidence of other intrinsic sleep disorders; stable therapy for pain; no therapy prescribed specifically for insomnia (subjects willing to discontinue medications prescribed for insomnia were eligible for the trial); stable pain medication regime (Table 2).

\section{Screening Procedure}

Subjects underwent a full physical examination, urinalysis, and blood work to rule out unstable medical illness, pregnancy, fibromyalgia, seizure disorder or evidence of active substance or alcohol abuse. The Mini International Neuropsychiatric Interview (MINI) and urine toxicology were used to rule out acute psychiatric and addiction problems. The subjects underwent one night of polysomnography (PSG) to rule out sleep disorders other than insomnia.

\section{Intake Pain Assessment}

To ensure the subject met inclusion criteria with respect to the pain condition (and to establish pretreatment values) the patient underwent a comprehensive pain assessment using the McGill Pain Index. Subjects also filled out a medication list that included specific doses and frequencies of use, the Multidimensional Pain Inventory, and a medical history including current pain management therapies. Once deemed to be stable (no changes in any treatments for the pain condition for the past 3 months) the subjects were instructed to avoid making changes to their pain management regimen (or the initiation of any medications) while in the study. In order to assess for compliance with this instruction, subjects were re-evaluated at the end of the CBT-I treatment phase using 1) the same questionnaires as completed at baseline and 2) a comprehensive study chart review. Change from the baseline treatment regime was coded in a zero-one fashion and percent of subjects exhibiting no change was assessed per group.

\section{Measures}

Sleep/pain diaries-This instrument allowed for the daily assessment of self reported sleep continuity and average pain intensity. Sleep continuity questions required that subjects record Time to Bed, Time Out of Bed and estimates of Sleep Latency (time in minutes from lights out to sleep onset) [SL]), Wake after Sleep Onset (total number of minutes spent awake from sleep onset to time out of bed [WASO]), and duration of Early Morning Awakening (time awake prior to planned wake time [EMA]). Subjects also recorded the number of awakenings they experienced per night (NWAK). Total Sleep Time [TST]) values were derived from the above values $(\mathrm{TST}=[$ Total Time in Bed $]-[\mathrm{SL}+\mathrm{WASO}+\mathrm{EMA}])$. Sleep Efficiency values were also derived from the above values ([Total Sleep Time / Total Time in Bed]*100).

All other variables (e.g., sleep quality, fatigue, stress, pain, alertness, concentration and mood) were expressed using a 0-10 scale, where 10 represented the most negative symptomatology. One variable (portion of day able to function), was reversed scored. Subjects were instructed to complete the sleep continuity portion of the diary first thing in the morning and the symptoms portion of the diary at the end of the day. Diaries were completed on a continuous basis (daily) beginning at study intake (pre treatment) and ending two weeks after the completion of the intervention phase of the study. Week eight diary assessment was used as the post treatment measure. 
Standardized questionnaires-Several instruments were administered at intake and sessions 1-8. The instruments used for this analysis included the: Insomnia Severity Index (ISI), Pain Disability Index (PDI), Multidimensional Pain Inventory (MPI) and the Beck Depression Inventory (BDI). The Epworth Sleepiness Scale (ESS), the Multidimensional Fatigue Index (MFI), and a symptom checklist were also administered on a weekly basis during treatment to assess iatrogenic effects or adverse events.

While the majority of the instruments noted above are common to insomnia research, the Multidimensional Pain Inventory and the Pain Disability Index are not and therefore need further description. The MPI is a 60-item self-report inventory designed to assess cognitive, behavioral, and affective response to pain. The MPI consists of 12 scales. Two of the scales (pain severity and pain interference) scales were used in the present analysis. The pain severity scale consists of three questions about pain intensity which are scored 0 to 6 . The sum score is divided by the number of questions to obtain the average scale score. An example of a question from this scale is "Rate the level of your pain at the present moment". The mean norm for this scale in chronic pain patients is $4.2 \pm 1.2(10,11)$. The interference scale assesses how pain interferes with activities of daily living, work, life and social functioning. The scale consists of 9 questions each of which are scored on a 0 to 6 scale. The sum score, as with the prior scale, is divided by the number of questions to obtain the average scale score. An example of a question from this scale is "In general, how much does your pain interfere with your dayto-day activities?" The mean norm for this scale in chronic pain patients is $4.0 \pm 1.5(10,11)$. The Pain Disability Index is a 7-question instrument that measures the degree that pain interferes with functioning. It is scored on a 0-10 numeric scale where zero means no disability at all, and a score of 10 signifies that all of normal activities have been totally disrupted or prevented by pain $(12,13)$.

\section{Treatment}

CBT-I-Subjects received eight weekly individual sessions of treatment which were delivered by a Masters prepared nurse therapist. The therapist's training included the following components: 1) Attended a 2.5 day seminar on diagnosing and treating sleep disorders, and the delivery of CBT-I. 2) Viewed a series of videotaped CBT-I sessions conducted by a licensed clinical psychologist who had experience conducting CBT-I in research and clinical venues. 3) Received weekly supervision (60-120 minutes per week) for the duration of the study by experienced therapist.

The eight weekly session protocol adhered to the regimen specified in our published treatment manual (14) and included 4 central components: Sleep Restriction Therapy, Stimulus Control Instructions, Sleep Hygiene Instructions, and one session of cognitive therapy (Figure 1).

Subject Adherence-This was assessed in two ways: 1) via the sleep diaries (reported Timeto-Bed or Time-out-of-Bed vs. the prescribed Time-to-Bed or Time-out-of-Bed) and by a mismatch between WASO and SL to reported Time-out-of-Bed during the night; 2) via actigraphy data (marker defined Time-to-Bed or Time-out-of-Bed and/or actigraphically assessed sleep onsets and offsets vs. prescribed Time-to-Bed or Time-out-of-Bed).

Discrepancies of $\geq 15$ minutes prompted the therapist to address this issue in session.

\section{Contact/measurement Control Condition}

Each subject randomized to this condition met with our nurse therapist once a week for a time period comparable to that required for CBT-I. During each session (45-90 minutes) the therapist and the subject reviewed the sleep/pain diary and BDI items which were above a scale score of 0 for the prior week. The items from the BDI served as topics for discussion given the rationale that life stress and depression are likely to perpetuate or intensify the patients' 
insomnia and pain severity. The review was interrogative only. No directed form of therapy was provided for pain, depression, or sleep disturbance.

\section{Data analysis}

Test for initial differences-Logistic Regression was performed to determine if baseline (collected at intake) characteristics of the subjects would predict group assignment. Age, gender, body mass index, race, educational level, occupational status, Pain Interference Scale score and Pain Severity Score from the MPI, total Beck Depression Inventory, and total Insomnia Severity Score were simultaneously entered. None of the variables were able to significantly predict group assignment. Nonetheless, we controlled for these baseline variables using the backward elimination procedure. For the six sleep outcomes and nine pain functioning variables, only age was significant for the model for TST (p-value $=0.046$ ). But to capture more information of covariates, any covariate with $\mathrm{p}$-value $<0.10$ was kept in the models for the analyses.

Management of missing data and multiple comparison-The impact of missing data was characterized by model estimates through two well-established missing data mechanisms: missing completely at random assumption (MCAR) and missing at random assumption (MAR) (15). The MCAR assumption was tested by modeling the statistical "missingness" of the patients' responses as a function of observed responses using logistic regression. If the results of logistic modeling show that missingness depends on the observed responses at baseline, then MCAR is inappropriate and Weighted Generalized Estimating Equations (WGEE) were used with weights estimated from the logistic model for missing data (15). Otherwise, missing data follows the MCAR model and the Generalized Estimating Equations (GEE) were used to model the differences from pre-to-post treatment between the two treatment conditions.

In the present study, there was no evidence to reject MCAR and therefore the GEE procedure was used to assess treatment effects for each of the outcomes. The analysis was conducted using the Genmod procedure in SAS. Pre-post treatment effects were modeled using the time by treatment interaction term.

The False discovery rate (FDR) was used to effectively control for Type I errors for multiple comparisons (16). The FDR approach controls for the false positive (or discovery) rate among the tests that are declared to be positive or statistically significant, providing a more relevant and accurate criterion for statistical significance and a more effective way for controlling inflated type I errors caused by multiple testing. For the current study, the number of tests used for applying FDR is based on the number of tests from all subscale analyses with respect to the time by treatment condition interaction within each domain examined. For example, when studying the sleep functioning domain, there are six tests, representing the six interaction terms for the models for each of the six outcomes. The FDR approach, therefore, was used to determine statistical significance for each test to ensure a family-wise overall rate of alpha $=$ 0.05 for the 6 tests. Note that covariates were not counted in determining the number of tests performed since they were not primary predictors.

\section{Significance testing}

Assessment of treatment response: In addition to the assessment of mean change, a responder/remitter analysis was conducted. Treatment response was defined based on the within subject meta-analytic norms published by Smith and colleagues (17) where a positive treatment response was defined as $43 \%$ improvement for SL, or $31 \%$ for NWAK, or $55 \%$ for WASO. Remission (achievement of normal sleep) was defined as an SL and WASO of less than 15 minutes. 
Calculation of effect sizes: In order to 1) provide a standardized metric of the observed effects and 2) allow for a comparison of the present study outcomes to the prior studies and to metaanalytic norms, between and within subject effect sizes ${ }^{\mathrm{ii}}$ were calculated for all the sleep continuity variables.

\section{Results}

\section{Subjects (sample acquisition and attrition)}

Forty-seven subjects with chronic non-malignant pain located in the spinal region (neck and back) gave consent during the screening phase of the study. Twenty-eight subjects met study eligibility criteria and were randomized $(\sim 2: 1)$ to either CBT-I or a contact/measurement control condition (19 CBT-I, 9 controls). Study eligible subjects were double-match randomized by a blinded third party until the $16^{\text {th }}$ subject was reached; at that point a stratification procedure according to gender, age and ethnicity occurred. The double matching procedure was used so that a larger sample of subjects could be exposed to the experimental condition.

Of the nineteen subjects not randomized, 8 were screen failures for other sleep disorders, 8 withdrew for personal reasons (no longer wished to participate), and 3 were not assigned to a treatment condition as the study had ended.

See Table 2 for subject characteristics.

\section{Subjects (sample characteristics $[\mathrm{n}=28]$ )}

The mean age of the subjects participating in this study was $48.7 \pm 10.7$ years of age. Seventyeight percent of the sample was female. The average ISI was $17.4 \pm 3.8$ and this corresponded to a mean baseline SL of $39 \pm 20.5$, WASO of $55.2 \pm 38.2$, NWAK of $3.4 \pm 2.3$, EMA of 27.8 \pm 23.8 , TST of $381.2 \pm 57.8$, and SE of $80 \pm 9.5 \%$. The average MPI severity score was $3.9 \pm$ 0.8 and the average MPI Interference score was $3.9 \pm 1$. The pain scores (out of a full scale of 7) represent moderate severity.

Of the 28 subjects enrolled into the treatment phase of the study, 18 suffered from low back pain, 9 from neck pain, and 1 from thoracic spinal pain. All subjects had a history of chronic pain for more than 6 months; $75 \%$ of the subjects had chronic pain for greater than 5 years. The site of pain did not change over the course of the study and the groups did not significantly differ with respect to the proportion of subjects that changed their treatment regimen during the treatment phase of the study (27\% change in CBT-I subjects and $20 \%$ change in the control condition [p >0.76]). Of the subjects that changed their medication regimens during treatment about $40 \%$ increased their analgesic doses, $40 \%$ decreased their analgesic doses, and about $10 \%$ changed medications though the doses were considered equipotent. To assess if improved sleep could have been associated with soporific pain medication effects that are usually exhibited by excessive daytime sleepiness, post treatment total score on the Insomnia Severity Scale was regressed on post treatment total score on the Epworth Sleepiness Scale. Severity of insomnia was found to significantly predict excessive daytime sleepiness $(F(1,19)=4.725$, p. $=.043)$. A positive relationship between insomnia score and excessive daytime sleepiness was found. The patients with lower scores on the insomnia scale also reported less excessive daytime sleepiness. It is unlikely that improved sleep was the result of soporific pain medications. For a listing of pain medications by group, please refer to Table 2 .

\footnotetext{
${ }^{i i}$ Between subject effect sizes = post Tx group differences in pooled standard deviation units Within subject effect sizes = pre-post change in pooled standard deviation units
} 


\section{Treatment response}

Mean Change in Sleep Continuity as Assessed with Sleep Diaries-After controlling for a family-wise type I error at the 0.05 level using the FDR method, subjects in the CBT-I group, as compared to the control group, exhibited significant pre-to-post improvement (Baseline vs. $8^{\text {th }}$ session). SL, WASO, NWAK, were significantly decreased (p. $=0.0003 ; 0.003 ; 0.008$ respectively) and SE was significantly $(\mathrm{p}=.002)$ increased. TST and EMA were not significantly changed $(\mathrm{p}=.14$ and 0.38 respectively). The group means for these variables did, however, have a trend in the appropriate direction (> TST and < EMA). Sleep Continuity as measured by the Insomnia Severity Index also showed significant improvement (p. $=0.0277)$.

Percent of subjects that exhibited treatment responses or achieved a SE of greater than $90 \%$-Fifteen of 19 subjects in the CBT-I group (78\%) and 2 of 9 subjects in the control group (22\%) were designated as treatment responders. Eight of 19 subjects in the CBT-I group (42\%) and 1 of 9 subjects in the control group (11\%) achieved normal sleep (remission).

Mean Change in Pain-Significant differences (p. $=0.0318)$ were evident for the Interference Scale from the MPI. The two groups did not differ on the sleep diary measure of average daily pain ( $\mathrm{p} .=0.6669)$, pain severity scale of the MPI $(\mathrm{p} .=0.2645)$, and the Pain Disability Index (p. =.0656).

Mean Change in Mood-Significant $(\mathrm{p} .=0.236)$ group differences were not seen on the Beck Depression Scale (Table 3 and Figure 3).

Effect Sizes-While descriptive in nature, rendering the current results in terms of effect sizes has, as noted above, the utility of 1) providing a standardized metric of the observed effects and 2) allowing for a comparison of the present study outcomes to the prior studies. Accordingly, between and within effect sizes are presented in Table 4 along with values from the prior studies (7-9) and the meta-analytic norms for Primary Insomnia (17). The data from all the studies are derived from sleep diary assessments of SL, WASO, and NWAK. The TST data from the present study, while based on sleep diary data, differ from the others in that it is a derived variable. Given these considerations, the data in Table 4 suggest that the present results are equal to, or better, than the prior pain studies and appear comparable to the metaanalytic norms for Primary Insomnia.

\section{Discussion}

In the present study the efficacy of CBT-I was assessed in patients with chronic pain. Pre-Post changes for sleep continuity, pain and mood were assessed across two groups; a CBT-I group $(n=19)$ and a contact/measurement control group $(n=9)$. Overall, with a family-wise type I error controlled at the 0.05 level by the FDR method, SL, WASO, and NWAK were significantly decreased and SE was significantly increased in the group that received CBT-I, while TST and EMA were not significantly changed. The significant findings for SL, WASO, NWAK and SE correspond to between-subject effect sizes of $1.44,1.51,0.57$ and 1.52 respectively. Seventyeight percent of the subjects who received CBT-I were designated as treatment responders (vs. $22 \%$ of the controls) and $42 \%$ were designated as in remission (vs. $11 \%$ of the controls). Confirmatory analysis using the Epworth Sleepiness Scale revealed that improved sleep was predictive of decreased daytime symptoms such as excessive daytime sleepiness. The groups did not significantly differ on the mood measures or sleep diary measures of pain. Limitations of this study include the small sample size, $25 \%$ attrition rate, and lack of objective sleep and 
pain measures. Although these limitations impact the strength of the study, the limitations are typical of behavioral intervention studies in patients with chronic pain.

\section{Why was CBT-I effective? Why were the sleep continuity effects large?}

The present results are consistent with the perspective that, when chronic, insomnia is indeed perpetuated by a common set of factors that function independently of precipitating events, or comorbidities. It may also be the case that several of the standard components CBT-I were especially effective because the factors targeted were not less, but instead were more operative than usual. While it will ultimately take a dismantling study to appropriately address this point of view, it stands to reason that the sleep restriction and stimulus control interventions may have been particularly effective in pain patients.

In the case of sleep restriction, it is generally thought to be effective because it serves to reverse the effects of "sleep extension", i.e., reverses the sleep homeostasis dysregulation that occurs with the expansion of sleep opportunity. This may be particularly relevant for insomnia comorbid with chronic pain as these patients may expand sleep opportunity not only in response to sleep loss but also as a means of managing pain.

In the case of stimulus control, it is generally thought to be effective because it serves to 1) establish a strong association between the bedroom and sleep via preventing non-sleep related behaviors from occurring in the sleep environment and by allowing for sleep to occur uniquely in association with the bedroom, 2) promote a sleep schedule that is regimented and yet still tied to the experience of "sleepiness", 3) allow for the counter-conditioning effects that come from the regular co-occurrence of sleep related stimuli and the physiology of sleepiness and sleep. Each of these factors may also be particularly relevant for insomnia comorbid with chronic pain. Pain patients may be subject to more stimulus "dyscontrol" than is normally the case as their pain may prompt them to seek sleep when not sleepy, to sleep in places other than the bedroom, and to engage in more non-sleep behaviors in the bedroom.

\section{Why were no significant effects observed for pain severity?}

One of the guiding hypotheses for the present study was that if sleep continuity was improved, patients would experience less pain. Physiologically this seems plausible as demonstrated by several sleep deprivation studies of healthy subjects reporting that sleep deprivation is hyperalgesic (18-20). It was recently demonstrated, for example, that sleep continuity disruption in healthy subjects impairs psychophysical measures of endogenous pain inhibitory capacity (20). It has also been shown, in longitudinal studies, that insomnia is an independent risk factor for development of chronic pain after acute injury, and the spread of pain from a regional disorder to a more widespread condition $(18,21,22)$.

The failure to resolve an acute post treatment effect of CBT-I on pain severity may be due to two main factors, 1) statistical power and 2) poor temporal resolution on phenomena that may require more than 8 weeks to unfold. With respect to the issue of power, our post treatment between group effect of CBT-I reducing MPI pain severity and diary average pain were 0.6 and 0.3 respectively. These effect sizes, although not statistically significant, would likely be resolved with a larger sample. In fact, our observed effects sizes on pain are comparable, if not better than the effects of cognitive-behavioral therapy for pain on pain severity (23).

Furthermore, the time course of when sleep-related improvements might be expected to yield their most pronounced effects on clinical pain remains unclear. While experimental studies of healthy young adults suggest that sleep disruption induced hyperalgesia normalizes quickly after recovery sleep, this may not be the case in patients with chronic pain who have demonstrated chronic sleep disruption. Also unclear is which dimensions of sleep continuity 
and architecture have the most robust influence on pain sensitivity in clinical populations. Several clinical trials testing CBT-I show that many aspects of sleep, particularly total sleep time continue to improve after treatment. This raises the distinct possibility that the effects of CBT-I on pain may be more robust during long-term follow up periods. We are currently in the process of analyzing our follow up data to evaluate this possibility. This said, the alternative possibility is that short and long term gains simply do not occur in a reliable fashion in chronic pain patients precisely because the tissue/structural/system damage is irreparable and no amount of sleep related "healing" can alter this scenario. If one accepts this proposition, it should be noted that this still leaves open the interesting possibility that improved sleep continuity may influence the healing processes (and thereby perceived pain) in acute trauma.

\section{What does it mean to show significantly reduced pain interference?}

Pain interference refers to the extent to which pain interferes with daily functioning and life satisfaction. Absent robust post-treatment changes in pain severity suggests that the insomnia was independently associated with the perceived impact of pain on daily functioning and life satisfaction and that these effects were reversed with the sleep continuity improvements that occurred in the subjects treated with CBT-I. One way this might be understood is akin to how patients sometime describe the effects of opioid analgesics. The pain may remain present or relatively constant, but it "seems to matter less." This distinction between pain and pain-related interference will require further investigation as it is quite possible that improvements in sleep quality may indirectly improve pain related outcomes via bolstering active coping resources. With respect to the potential clinical significance of the reductions in pain-related interference it would seem that the consequences of insomnia on daytime function pale in comparison to those of chronic pain. This may not, however, be the case. The effect size for pain interference was moderate to large $(0.71)$ and the change in the mean scale score was of a magnitude deemed clinically relevant for pain interventions. That is, in the present study there was a 1.1 decrease on a scale where $\geq 0.6$ point decreases are considered clinically relevant $(24,25)$.

In summary, the sleep continuity results of this study provide further evidence that CBT-I can be effectively applied to patients with chronic pain. Further, when the present results are viewed alongside the other insomnia and chronic pain RCTs and the meta-analytic norms for Primary Insomnia, it appears that the within-subject effect sizes equal, or exceed, these "benchmarks." This high level of comparability not only serves to increase confidence in the broad applicability of CBT-I but also calls into question the validity and/or utility of the nosologic distinction between Primary and Secondary Insomnia. That is, if the treatment indications and outcomes are the same then the different diagnostic labels have only limited usefulness and only serve to denote a potential precipitant and/or a current comorbidity. This being the case, the current term "insomnia comorbid with chronic pain" (vs. Secondary Insomnia or Insomnia Secondary to Chronic Pain) may indeed be more apropos.

Future studies are needed to determine 1) the durability of acute treatment effects, 2) what components of CBT-I work best (and what components are unnecessary) for insomnia comorbid with chronic pain, 3) what components might be added to produce greater clinical effects and 4) how CBT-I affects medico-economic outcomes like health care utilization and work function.

\section{Acknowledgments}

Funding/support: This research is supported by NINR NR5R21NR009080-02 The authors would like to acknowledge support from the University of Rochester, School of Nursing Center for Research and Evidence Based Practice data management team and Xin Tu, PhD, Professor and Associate Chair of the University of Rochester, Department of Biostatistics. 


\section{References}

1. Lichstein KL. Secondary insomnia: A myth dismissed. Sleep Med Rev 2006 Feb;10(1):3-5. [PubMed: 16380276]

2. McCrae CS, Lichstein KL. Secondary insomnia: Diagnostic challenges and intervention opportunities. Sleep Med Rev 2001 Feb;5(1):47-61. [PubMed: 12531044]

3. Spielman A. Assessment of insomnia. Clinical Psychology Review 1986;6:11-25.

4. Spielman AJ, Caruso LS, Glovinsky PB. A behavioral perspective on insomnia treatment. Psychiatr Clin North Am 1987 Dec;10(4):541-53. [PubMed: 3332317]

5. National Institutes of Health. National institutes of health state of the science conference statement on manifestations and management of chronic insomnia in adults, june 13-15, 2005. Sleep 2005 Sep 1;28 (9):1049-57. [PubMed: 16268373]

6. Roth T, Franklin M, Bramley TJ. The state of insomnia and emerging trends. Am J Manag Care 2007 Nov;13(5 Suppl):S117-20. [PubMed: 18041871]

7. Rybarczyk B, Stepanski E, Fogg L, Lopez M, Barry P, Davis A. A placebo-controlled test of cognitivebehavioral therapy for comorbid insomnia in older adults. J Consult Clin Psychol 2005 Dec;73(6): 1164-74. [PubMed: 16392989]

8. Currie SR, Wilson KG, Pontefract AJ, deLaplante L. Cognitive-behavioral treatment of insomnia secondary to chronic pain. J Consult Clin Psychol 2000 Jun;68(3):407-16. [PubMed: 10883557]

9. Edinger JD, Wohlgemuth WK, Krystal AD, Rice JR. Behavioral insomnia therapy for fibromyalgia patients: A randomized clinical trial. Arch Intern Med 2005 Nov 28;165(21):2527-35. [PubMed: 16314551]

10. Kerns RD, Turk DC, Rudy TE. The west haven-yale multidimensional pain inventory (WHYMPI). Pain 1985 Dec;23(4):345-56. [PubMed: 4088697]

11. Kerns RD, Finn P, Haythornthwaite J. Self-monitored pain intensity: Psychometric properties and clinical utility. J Behav Med 1988 Feb;11(1):71-82. [PubMed: 3367373]

12. Changulani M, Shaju A. Evaluation of responsiveness of oswestry low back pain disability index. Arch Orthop Trauma Surg. 2008 Jun 3;

13. Chibnall JT, Tait RC. The pain disability index: Factor structure and normative data. Arch Phys Med Rehabil 1994 Oct;75(10):1082-6. [PubMed: 7944912]

14. Perlis, ML.; Jungquist, CR.; Smith, MT.; Posner, D. Cognitive behavioral treatment of insomnia: A session by session guide. New York: Springer Science+Business Media, Inc.; 2005.

15. Kowalski, J.; Tu, XM. Modern Applied U Statistics. New York: Wiley; 2007. Models for clustered data.

16. Benjamini Y, Hochberg Y. Controlling the false discovery rate: A practical and powerful approach to multiple testing. J R Stat Soc Ser B-Methodol 1995;57(1):289-300.

17. Smith MT, Perlis ML, Park A, Smith MS, Pennington J, Giles DE, et al. Comparative meta-analysis of pharmacotherapy and behavior therapy for persistent insomnia. Am J Psychiatry 2002 Jan; 159(1): 5-11. [PubMed: 11772681]

18. Smith MT, Klick B, Kozachik S, Edwards RE, Holavanahalli R, Wiechman S, et al. Sleep onset insomnia symptoms during hospitalization for major burn injury predict chronic pain. Pain 2008 Sep 15;138(3):497-506. [PubMed: 18362052]

19. Roehrs T, Hyde M, Blaisdell B, Greenwald M, Roth T. Sleep loss and REM sleep loss are hyperalgesic. Sleep 2006 Feb 1;29(2):145-51. [PubMed: 16494081]

20. Smith MT, Edwards RR, McCann UD, Haythornthwaite JA. The effects of sleep deprivation on pain inhibition and spontaneous pain in women. Sleep 2007 Apr 1;30(4):494-505. [PubMed: 17520794]

21. Mikkelsson M, Sourander A, Salminen JJ, Kautiainen H, Piha J. Widespread pain and neck pain in schoolchildren. A prospective one-year follow-up study. Acta Paediatr 1999 Oct;88(10):1119-24. [PubMed: 10565460]

22. Mikkelsson M. One year outcome of preadolescents with fibromyalgia. J Rheumatol 1999 Mar;26 (3):674-82. [PubMed: 10090181] 
23. Morley S, Eccleston C, Williams A. Systematic review and meta-analysis of randomized controlled trials of cognitive behaviour therapy and behaviour therapy for chronic pain in adults, excluding headache. Pain 1999 Mar;80(12):1-13. [PubMed: 10204712]

24. Dworkin RH, Turk DC, Wyrwich KW, Beaton D, Cleeland CS, Farrar JT, et al. Interpreting the clinical importance of treatment outcomes in chronic pain clinical trials: IMMPACT recommendations. J Pain 2008 Feb;9(2):105-21. [PubMed: 18055266]

25. Dworkin RH, Turk DC, Farrar JT, Haythornthwaite JA, Jensen MP, Katz NP, et al. Core outcome measures for chronic pain clinical trials: IMMPACT recommendations. Pain 2005 Jan;113(12):919. [PubMed: 15621359] 


\section{CBT of Insomnia Session by Session Treatment Protocol}

Session 1 - 45-60 minutes (case review). The therapist reviews the case details with the patient. No active treatment is provided. Sleep/pain diaries continue.

Session $2-45-90$ minutes (sleep restriction and stimulus control therapy). Baseline sleep diary data are reviewed from previous 1-2 week interval. This information sets the parameters for sleep restriction therapy and is used to guide the patient toward the treatment to be prescribed. Our standard approach is interactive/didactic. During this session, the clinician explains in detail the rationale using the Spielman Model, and the procedures from sleep restriction and stimulus control therapy.

Session 3 - 30-45 minutes (sleep hygiene and sleep restriction therapy adjustments). As with all sessions, sleep diary data are reviewed, continuity variables calculated, and data charted in bar graph format. The upward titration by adding 15 minutes to prescribed time in bed is initiated if the sleep efficiency was greater than $90 \%$. Sleep hygiene instructions are reviewed.

Session 4 \& 5 - 30-45 minutes (sleep restriction therapy adjustments). Sleep diary data are reviewed and charted. Upward titration is continued as long as sleep efficiency met at least $90 \%$ for the previous week.

Session 6 - 45-60 minutes (sleep restriction therapy adjustments and cognitive restructuring). Upward titration is continued and the cognitive component of the treatment regimen is initiated. Barlow-style approach to "decatastrophization" is used to address the perception of dire consequences of sleep loss.

Session 7 - 30-45 minutes (sleep restriction therapy adjustments). Sleep diary data are reviewed and charted. Upward titration continues.

Session 8 - 30-45 minutes (sleep restriction therapy adjustments and relapse prevention). Relapse prevention issues are reviewed. Patient is instructed to continue upward titration of time in bed until sleep efficiency drops and stays below $90 \%$.

Figure 1. Session by Session Description of CBT-I 


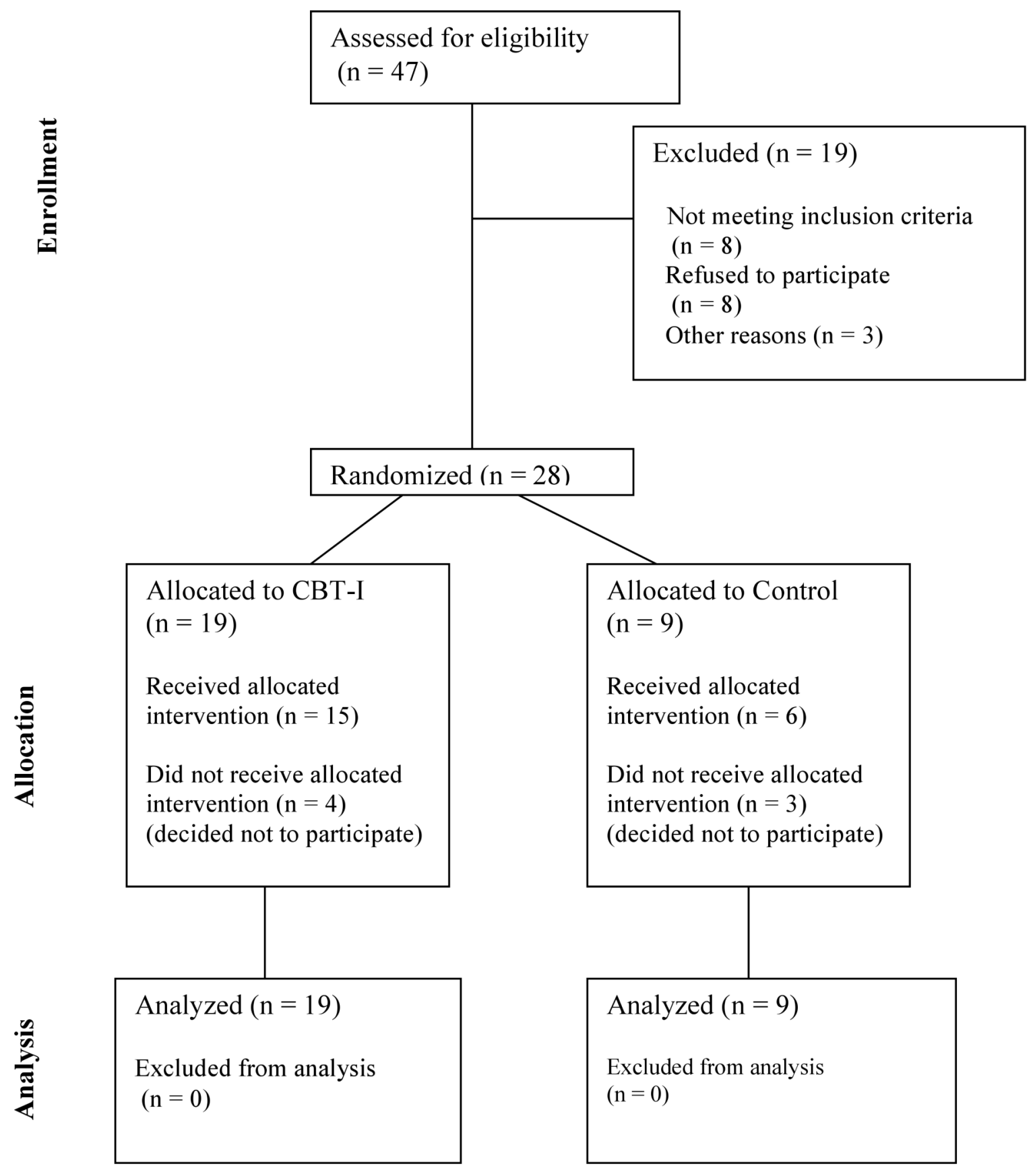

Figure 2.

CONSORT Diagram 


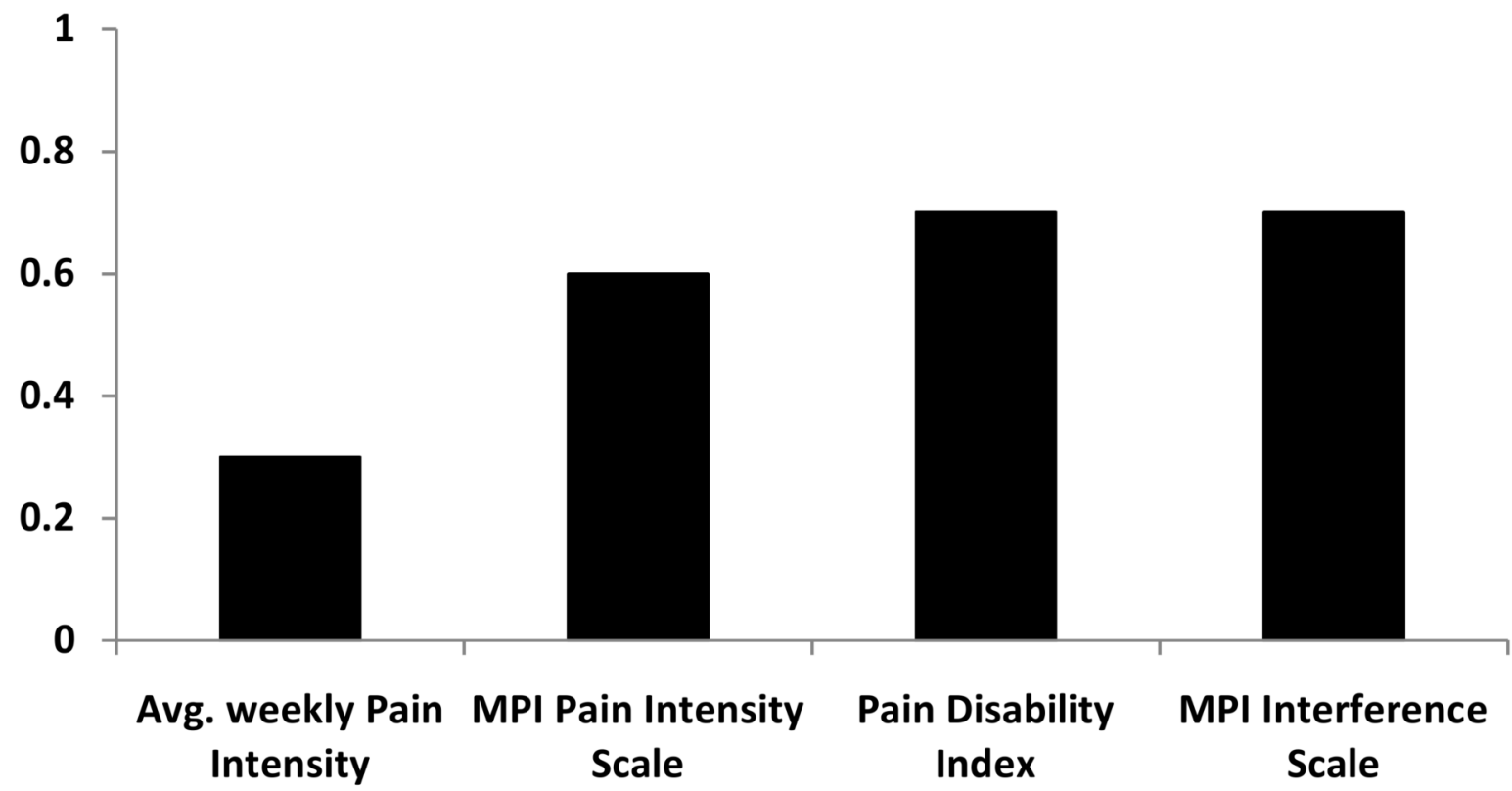

Figure 3.

Pre-Post Between Group Effect Sizes for Measures of Pain 


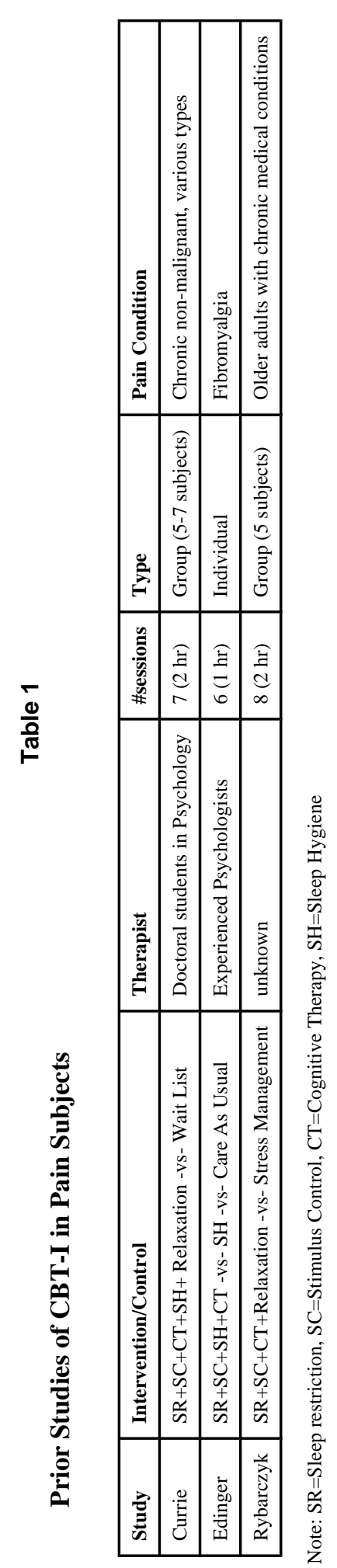

Sleep Med. Author manuscript; available in PMC 2011 March 1. 


\section{Group Characteristics}

Table 2

\begin{tabular}{|c|c|c|}
\hline Variable & CBT Group $(n=19)$ & Controls $(n=9)$ \\
\hline Age, mean(SD) & $52(9.9)$ & $43(10.7)$ \\
\hline Gender F:M & $5: 1$ & $8: 1$ \\
\hline BMI & $27(4)$ & $27(5.2)$ \\
\hline \multicolumn{3}{|l|}{ Education } \\
\hline High School & 5 & 5 \\
\hline Associates & 9 & 2 \\
\hline Bachelors & 4 & 1 \\
\hline Graduate & 2 & 1 \\
\hline \multicolumn{3}{|l|}{ Race } \\
\hline White & 17 & 7 \\
\hline African American & 2 & 2 \\
\hline \multicolumn{3}{|l|}{ Occupation } \\
\hline Disabled & 4 & 3 \\
\hline Part Time & 4 & 3 \\
\hline Full Time & 11 & 3 \\
\hline Retired & 3 & 0 \\
\hline \multicolumn{3}{|l|}{ Sleep } \\
\hline Insomnia Severity Index (ISI) (range 0-28) & $17(3)$ & $19(3)$ \\
\hline \multicolumn{3}{|l|}{ Mental Health } \\
\hline Beck Depression Inventory (BDI) (range 0-63) & $12.7(6)$ & $11.8(9)$ \\
\hline \multicolumn{3}{|l|}{ Pain } \\
\hline MPI - Severity Scale (range 0-7) & $3.8(1)$ & $4.3(0)$ \\
\hline MPI - Interference Scale (range 0-7) & $3.9(0)$ & $3.9(1)$ \\
\hline \multicolumn{3}{|l|}{ Pain Medication Use (\% of subjects taking) } \\
\hline Opioid & $26 \%$ & $33 \%$ \\
\hline Tramadol & $0 \%$ & $22 \%$ \\
\hline Antidepressants & $52 \%$ & $22 \%$ \\
\hline NSAIDS & $63 \%$ & $22 \%$ \\
\hline Anticonvulsant & $21 \%$ & $22 \%$ \\
\hline Muscle relaxants & $10 \%$ & $22 \%$ \\
\hline
\end{tabular}

Clinically relevant thresholds: ISI 15-21= insomnia of moderate severity; BDI 14-19= mild depression 
Table 3

Primary Outcomes for Sleep and Pain Variables

\begin{tabular}{|c|c|c|c|c|}
\hline Measure & Group & Pre Treatment Mean & Post Treatment Mean & Mean Diff \\
\hline \multirow[t]{2}{*}{ Sleep Latency } & CBT-I & $37 \pm 18$ & $9 \pm 4$ & $-27^{*}$ \\
\hline & Control & $45 \pm 25$ & $44 \pm 27$ & 0 \\
\hline \multirow[t]{2}{*}{ Wake after Sleep Onset } & CBT-I & $58 \pm 44$ & $12 \pm 8$ & $-48^{*}$ \\
\hline & Control & $49 \pm 44$ & $44 \pm 32$ & -4 \\
\hline \multirow[t]{2}{*}{ Number of Awakenings } & CBT-I & $4 \pm 3$ & $2 \pm 1$ & $-2^{*}$ \\
\hline & Control & $3 \pm 1$ & $2 \pm 1$ & -1 \\
\hline \multirow[t]{2}{*}{ Early Morning Awakenings } & CBT-I & $25 \pm 22$ & $5 \pm 6$ & -20 \\
\hline & Control & $32 \pm 26$ & $20 \pm 24$ & -12 \\
\hline \multirow[t]{2}{*}{ Total Sleep Time } & CBT-I & $359 \pm 64$ & $408 \pm 35$ & 49 \\
\hline & Control & $343 \pm 66$ & $352 \pm 73$ & 9 \\
\hline \multirow[t]{2}{*}{ Sleep Efficiency } & CBT-I & $75 \pm 13$ & $94 \pm 3$ & $19^{*}$ \\
\hline & Control & $73 \pm 11$ & $76 \pm 16$ & 3 \\
\hline \multirow[t]{2}{*}{ Insomnia Severity Index } & CBT-I & $17 \pm 4$ & $4 \pm 4$ & $-13^{*}$ \\
\hline & Control & $19 \pm 3$ & $13 \pm 6$ & -6 \\
\hline \multirow[t]{2}{*}{ MPI Interference Scale } & CBT-I & $3.9 \pm 0.8$ & $2.7 \pm 1.5$ & $-1.1^{*}$ \\
\hline & Control & $3.9 \pm 1.4$ & $3.7 \pm 1.5$ & -0.2 \\
\hline \multirow[t]{2}{*}{ MPI Pain Severity Scale } & CBT-I & $3.7 \pm 1.0$ & $2.2 \pm 1.9$ & -1.5 \\
\hline & Control & $4.3 \pm 0.5$ & $3.6 \pm 1.3$ & -0.7 \\
\hline \multirow[t]{2}{*}{ Pain Disability Index } & CBT-I & $28 \pm 13$ & $14 \pm 16$ & -13 \\
\hline & Control & $33 \pm 15$ & $30 \pm 13$ & -3 \\
\hline \multirow[t]{2}{*}{ Avg. Daily Pain } & CBT-I & $4.7 \pm 1.3$ & $3.8 \pm 2.9$ & -0.8 \\
\hline & Control & $4.9 \pm 1.3$ & $4.6 \pm 2.6$ & -0.3 \\
\hline \multirow[t]{2}{*}{ Beck Depression Inventory } & CBT-I & $13 \pm 6$ & $2 \pm 3$ & -11 \\
\hline & Control & $12 \pm 9$ & $6 \pm 6$ & -6 \\
\hline
\end{tabular}

* statistically significant group by time differences; within and between effect size are in tables 4 \& figure 3. 


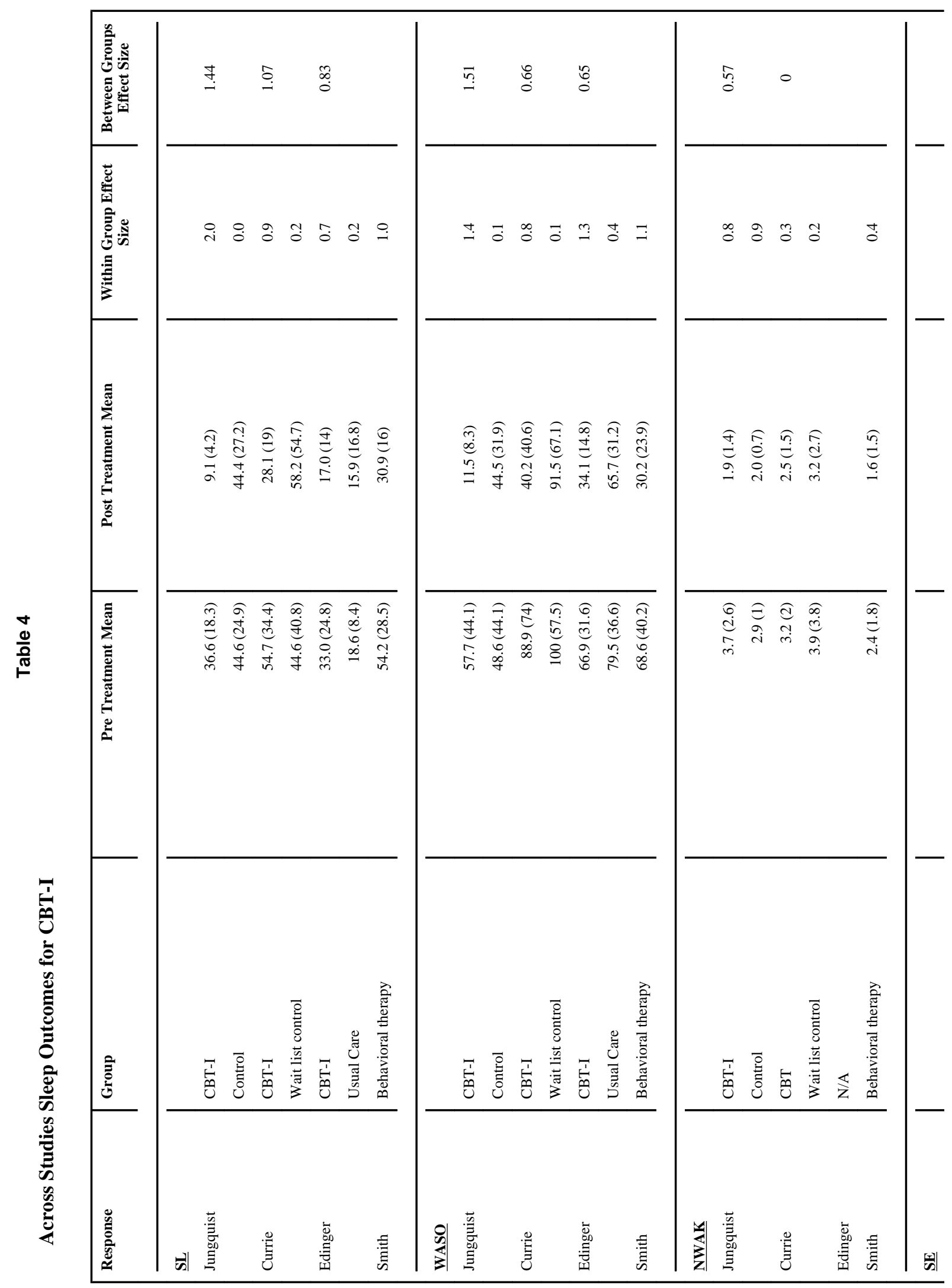

Sleep Med. Author manuscript; available in PMC 2011 March 1. 


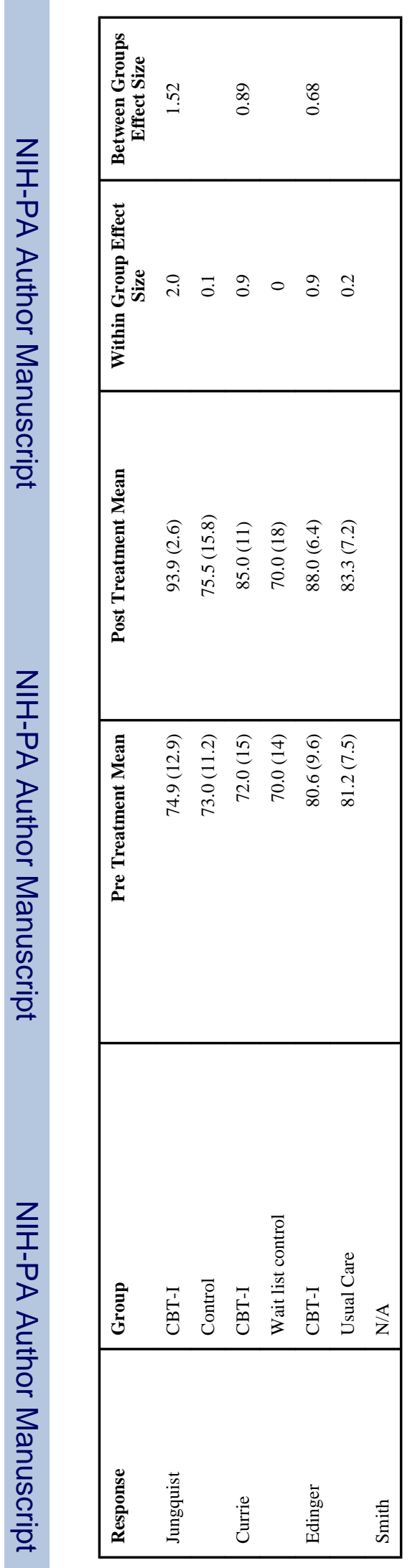

Sleep Med. Author manuscript; available in PMC 2011 March 1. 